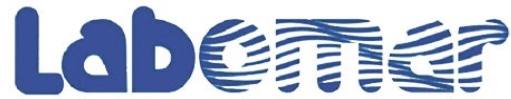

Arquivos de Ciências do Mar

\section{VARIAĢÃO DA LINHA DE COSTA NO LITORAL LESTE DO ESTADO DO GEARÁ: O CASO DA RESEX DA PRAINHA DO CANTO VERDE}

\section{Shoreline variation in the east coast of Ceará state: the case of Resex of Prainha do Canto Verde}

\author{
Eduardo Lacerda Barros ${ }^{1 *}$, Renan Gonçalves Pinheiro Guerra ${ }^{2}$, Rodrigo Passos Fernandes ${ }^{3}$ \\ ${ }^{1}$ Instituto de Ciências do Mar (Labomar), Universidade Federal do Ceará (UFC). E-mail: eduardo.Igco@gmail.com \\ ${ }^{2}$ Célula de Conservação da Diversidade Biológica (Cedib/Cobio), Secretaria do Meio Ambiente do \\ Estado do Ceará (Sema). E-mail: renan.Igco@gmail.com \\ ${ }^{3}$ Graduação em Ciências Ambientais, Instituto de Ciências do Mar (Labomar), Universidade Federal do Ceará (UFC) \\ E-mail: rodrigowalking2@gmail.com \\ *Autor para correspondência
}

\section{RESUMO}

A zona costeira, área de extrema dinamicidade devido à interação entre os sistemas marinhos e continentais e aos também fenômenos climáticos, tem passado por consequentes alterações em decorrência das mudanças climáticas cada vez mais recorrentes e severas, implicando diretamente na linha de costa, seja na forma de erosão, seja na progradação. Dessa forma, utilizando-se das metodologias associadas às técnicas de geoprocessamento, foi possível verificar que nos últimos 13 anos o trecho de praia que compreende a Resex da Prainha do Canto Verde, localizada no litoral leste do Ceará, apresentou taxas de erosão que variam entre $-4,14 \mathrm{~m} /$ ano e $-2,33 \mathrm{~m} /$ ano (EPR) e de $-3,66 \mathrm{~m} /$ ano a $-1,77 \mathrm{~m} /$ ano (LRR). Em termos totais, isso representa um recuo da linha de costa que variou entre pouco mais de $50 \mathrm{~m}$, no máximo, e mínimo de $23 \mathrm{~m}$ de acordo com as estatísticas utilizadas pela extensão DSAS.

Palavras-chave: erosão costeira, linha de costa, gerenciamento costeiro.

\section{ABSTRACT}

The coastal zone, an area of extreme dynamism due to the interaction between marine and continental systems, also subject to climatic phenomena, has been undergoing consequent changes due to increasingly recurrent and severe climate changes and this has directly implied the shoreline,

Recebido em: $13 / 11 / 2019$

Aprovado em: 26/06/2020

Publicado em: 30/01/2021 
either in the form of erosion or progradation. Thus, using methodologies associated with geoprocessing techniques it was possible to verify that in the last 13 years the beach stretch comprising the Resex of Prainha do Canto Verde, located on the east coast of Ceará, presented erosion rates ranging from $-4,14 \mathrm{~m} / \mathrm{yr}$ and $-2.33 \mathrm{~m} / \mathrm{yr}$ (EPR) and from $-3.66 \mathrm{~m} / \mathrm{yr}$ to $-1.77 \mathrm{~m} / \mathrm{yr}(\mathrm{LRR})$, in total terms this represents a shoreline retreat that ranged from slightly more than $50 \mathrm{~m}$, maximum and minimum 23 m according to the statistics used by the DSAS extension.

Keywords: coastal erosion, shoreline, coastal management.

\section{INTRODUÇÃO}

A zona costeira (ZC), área complexa devido à sua extrema dinamicidade, torna-se sensível a qualquer modificação de ordem natural relacionada à interação dos ambientes marinhos, continentais e climáticos e ainda é, cada vez mais, atingida pela pressão antrópica. Aproximadamente um quarto da população nacional reside em municípios litorâneos e isso está claramente relacionado ao potencial estratégico desse ambiente para o desenvolvimento de atividades que lhe são características, tendo como principais forças motrizes as atividades portuárias, industriais, petrolíferas e extrativistas, além do turismo.

Portanto, tais atividades inerentes a esse ambiente podem ser diretamente impactadas pela constante modificação da linha de costa (LC) ao longo do tempo, linha essa considerada como trecho de transição entre o continente e o oceano e que tem a sua posição condicionada pela atuação de processos atuantes na ZC, como ondas, correntes, marés e ação eólica. Devemos considerar também a atuação de eventos extremos (e.i. ressacas) atuantes com mais regularidade e intensidade durante o primeiro semestre na região Nordeste do país e que estão cada vez mais sujeitos às modificações ocasionadas pelas mudanças climáticas globais.

Nesse cenário de crescimento da ocupação voltada diretamente para o litoral, não devemos descartar os riscos associados à erosão costeira e as modificações na linha de costa que, por ventura, possam atingir o patrimônio edificado e, assim, serem revertidos em prejuízos econômicos para a sociedade local e para o Estado. Diante disso, o objetivo deste trabalho foi o de analisar a variação da linha de costa entre os anos de 2003 e 2016 em um trecho do município de Beberibe que compreende a Reserva Extrativista da Prainha do Canto Verde.

\section{Localização da área de estudo}

A área de estudo encontra-se localizada dentro dos limites da Reserva Extrativista da Prainha do Canto Verde, localizada no município de Beberibe, litoral leste do estado do Ceará. A 120 km de distância da capital Fortaleza, a Prainha do Canto Verde faz limite com o Oceano Atlântico ao norte, com os municípios de Palhano, Russas e Morada Nova ao sul, com os municípios de Aracati e Fortim a leste e com os municípios de Ocara e Cascavel a oeste (Figura 1 e Tabela I). O litoral do município possui $54 \mathrm{~km}$ de extensão e é delimitado pela planície fluviomarinha dos rios Choró, a oeste, e Pirangi, a leste (Costa, 2016). De acordo com Pontes e Silva (2011) e Mendonça (2012), a Resex está situada em uma área de faixa litorânea com a presença de campos de dunas fixas e móveis, lagoas temporárias, pós-praia, depressão interdunar, afloramentos de dunas antigas e da formação barreiras e planície fluviolagunar. 
Esse trecho do litoral cearense apresenta regime de maré semidiurna, do tipo mesomaré, com amplitudes que chegam a atingir 3,3 m. As ondas são provenientes dos quadrantes E-ESE e N-NE e estas últimas são características de eventos de alta energia (e.i. ondas de ressaca), configurando as ondas do tipo sea e swell (Maia, 1998; Morais et al., 2006; Guerra, 2018).

Figura 1 - Área de monitoramento na Prainha do Canto Verde, em Beberibe, Ceará

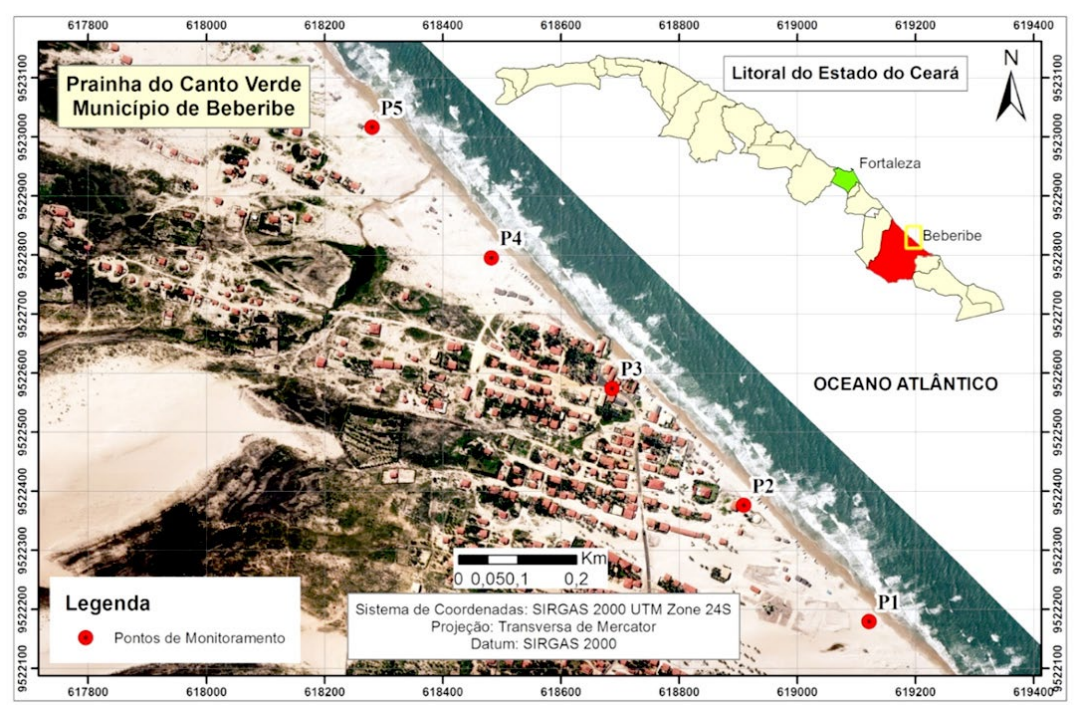

Fonte: os autores.

Tabela I - Síntese de informações sobre a área de estudo e suas características

\begin{tabular}{ccccc}
\hline Praia monitorada & $\begin{array}{c}\text { Extensão da linha de } \\
\text { costa do município }\end{array}$ & $\begin{array}{c}\text { Extensão da linha de } \\
\text { costa analisada }\end{array}$ & $\begin{array}{c}\text { Direção da linha } \\
\text { de costa }\end{array}$ & $\begin{array}{c}\text { Tipo de exposição } \\
\text { da linha de costa }\end{array}$ \\
\hline $\begin{array}{c}\text { Prainha do Canto } \\
\text { Verde }\end{array}$ & $54 \mathrm{~km}$ & $\begin{array}{c}1,225 \mathrm{~km} \\
(2,2 \% \text { do litoral })\end{array}$ & NW-SE & Exposta \\
\hline
\end{tabular}

Fonte: os autores.

\section{MATERIAIS E MÉTODOS}

As etapas metodológicas aplicadas neste trabalho foram divididas em três fases. Primeiramente foi realizado um levantamento prévio de informações sobre a área de estudo; a segunda fase está diretamente relacionada ao uso de técnicas de geoprocessamento para uma primeira análise da situação relativa à erosão costeira na área de estudo aqui proposta; e a última fase consistiu na interpretação dos resultados referentes à variação da linha de costa (LC) e na busca pelos possíveis fatores que condicionam a erosão no local.

Para se determinar o trecho analisado, buscamos inicialmente fazer um levantamento do estado da arte referente às temáticas da erosão costeira em artigos, periódicos e demais ferramentas de busca. Foi realizada também uma consulta ao acervo do Laboratório de Geologia e Geomorfologia Costeira e Oceânica (LGCO), da Universidade Estadual do Ceará (Uece), e do Instituto de Ciências do Mar (Labomar), da Universidade Federal do Ceará (UFC). As imagens de satélite empregadas para a extração das LC foram obtidas por meio do software Google Earth Pro e, posteriormente, georreferenciadas utilizando como base a ortofotocarta obtida na Superintendência Estadual do Meio Ambiente do Ceará (Semace). 
A segunda etapa utilizou-se de técnicas de geoprocessamento para obtenção das taxas de erosão e progradação da LC e, para isso, foi empregada a extensão Digital Shoreline Analysis System (DSAS), na versão 4.2, utilizada no ArcGIS 10.3. A extensão permite uma análise estatística das tendências erosivas e progradacionais de determinado local fazendo-se uso de uma série histórica de dados obtidos, seja por campo, seja por imagem de satélite. Foram aplicados para este estudo os métodos End Point Rate (EPR), que considera as linhas extremas de um determinado intervalo de tempo analisado, e o Linear Regression Rate (LRR), que se baseia em todos os dados referentes às linhas de costa utilizadas para a análise (Thieler et al., 2009).

A delimitação da LC pode ser feita de modo in situ, ou seja, em campo, ou por meio da utilização de uma série de indicadores, aqui considerados como geoindicadores. Tal marcador morfológico que limita o ambiente terrestre do marinho já foi bastante discutido ao longo da literatura internacional e nacional, como em Crowell, Leatherman e Buckley (1993), Leatherman (2003), Boak e Turner (2005), Baptista et al. (2011), Muehe (2014), Barros (2018) e Guerra (2018). Para este estudo, foram usados os geoindicadores propostos por Crowell, Leatherman e Buckley (1993) e Boak e Turner (2005). As características da área de estudo, as imagens utilizadas e os transectos estabelecidos pelo DSAS encontram-se detalhados na Tabela II e Figura 2.

Tabela II - Síntese de informações sobre a obtenção das linhas de costa e transectos estabelecidos

\begin{tabular}{ccccccc}
\hline $\begin{array}{c}\text { Praia } \\
\text { monitorada }\end{array}$ & Imagem & $\begin{array}{c}\text { Escala } \\
\text { temporal }\end{array}$ & $\begin{array}{c}\text { Resolução } \\
\text { espacial }\end{array}$ & $\begin{array}{c}\text { Número de } \\
\text { transectos }\end{array}$ & $\begin{array}{c}\text { Extensão dos } \\
\text { transectos } \\
(\mathbf{m})\end{array}$ & $\begin{array}{c}\text { Espaçamento entre } \\
\text { os transectos (m) }\end{array}$ \\
\hline $\begin{array}{c}\text { Prainha do } \\
\text { Canto Verde }\end{array}$ & $\begin{array}{c}\text { Quickbird } \\
(2003,2008,2016) \\
\text { Ortofoto } \\
(2014)\end{array}$ & 13 anos & $0,61 \mathrm{~m}$ & & & \\
& & $0,35 \mathrm{~m}$ & 49 & & 250 \\
\end{tabular}

Fonte: os autores.

Figura 2 - Área de estudo com os transectos estabelecidos pelo DSAS e linhas de costa utilizadas

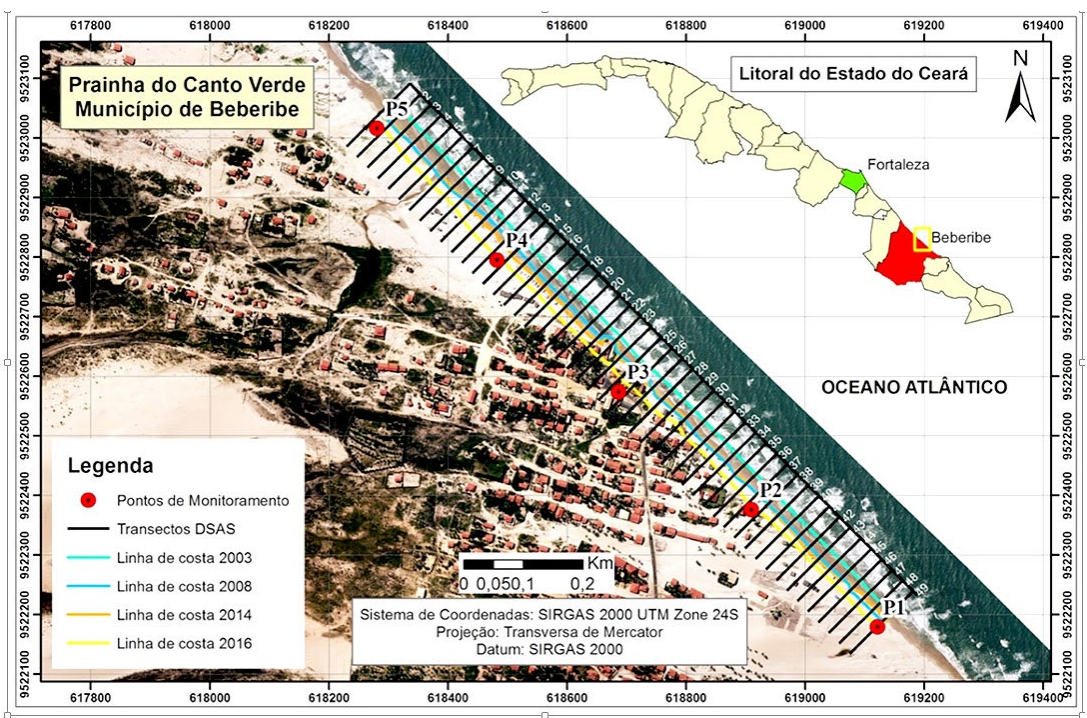

Fonte: os autores 


\section{RESULTADOS E DISCUSSÃO}

Podemos entender por erosão costeira como um processo decorrente da redução do balanço sedimentar que, por sua vez, diminui a largura da praia e provoca a perda do volume de sedimentos, além de uma série de desequilíbrios ambientais em decorrência desse deficit (Souza et al., 2005; Lins-de-Barros, 2005).

Portanto, a erosão é um processo natural que costuma ocorrer em diferentes escalas temporais e espaciais, sejam elas de caráter local, sejam de caráter global. De acordo com Morton (1979) e Bird (2008), o processo erosivo presente ao longo do litoral não se manifesta apenas em caráter local, mas também se trata de um processo que ocorre globalmente, podendo-se estimar que aproximadamente $70 \%$ das costas arenosas estão à mercê da intensificação de processos erosivos. Como fatores responsáveis por essas alterações no ambiente litorâneo, podemos destacar a influência de questões naturais, como o clima, a geologia e a geomorfologia, e a influência de fatores antrópicos, como a ocupação irregular de áreas fontes de sedimentos.

Segundo Farinaccio (2008), os impactos da erosão costeira vão desde a perda do valor imobiliário de edificações, o comprometimento de atividades turísticas, os gastos para recuperação dos locais atingidos até impactos com a redução da largura de praias e desequilíbrio de habitats naturais, como manguezais e campos de dunas. Tais impactos podem ser facilmente observados ao longo do litoral brasileiro há décadas, tornando-se um dos principais problemas ambientais e sociais do nosso território (Morais et al., 2018).

Nesse contexto, de análise da LC, os impactos da erosão costeira sobre essa faixa de transição podem ser tanto físicos como ecossistêmicos e sociais. Tal fato torna-se ainda mais agravante quando há um avançado do processo de ocupação dessas áreas, onde os danos se tornam potencializados (Farinaccio, 2008; Barros, 2018).

Em praias onde as feições são originadas pela interação de fluxos marinhos e fluviais, as alterações impostas pelo regime climático também colaboram diretamente na configuração da linha de costa e de áreas adjacentes (Morais et al., 2008, 2018). No caso de praias modificadas por marés, o gradiente de declividade da praia potencializa a amplitude dos processos hidrodinâmicos que atuam na remobilização de sedimentos e pode não só influenciar o fornecimento de sedimentos, mas também a retirada de material, o que, consequentemente, configura-se como erosão (Pinheiro; Marais \& Maia, 2016).

Dessa forma, faz-se necessário o entendimento da atuação em conjunto de fatores naturais e antrópicos na modificação do ambiente praial como forma de melhorar o nível de resposta sobre as causas da erosão e assim evitar possíveis danos futuros às estruturas construídas ao longo do litoral (Dias, 1990; Muehe, 2001; Borges et al., 2009; Muehe et al., 2014).

Assim, destacamos o litoral leste do estado do Ceará, no qual várias praias passam por esse processo, sendo uma delas a Prainha do Canto Verde. Localizada na reserva extrativista de mesmo nome, nos últimos 13 anos vem apresentando sérios problemas relativos aos danos em estruturas construídas muito próximas da linha de costa, assim como outras praias próximas, como Parajuru, também em Beberibe, Caponga, em Cascavel, Iguape, em Aquiraz, Barreiras da Sereia e Barrinha, em Icapuí. Todas têm passado por modificações de curto prazo dadas as suas condições ambientais.

A Figura 3 apresenta as taxas de variação da linha de costa para o trecho analisado segundo os cálculos obtidos com o DSAS, comparando os métodos estatísticos EPR e LRR. Foi possível observar para ambos os métodos que durante o intervalo de 13 anos houve 
somente erosão para esse trecho do litoral de Beberibe e não houve qualquer indício de progradação. A confiabilidade dos resultados é apresentada na Figura 4, que apresenta o gráfico de correlação entre os métodos estatísticos, mostrando uma perfeita correlação com $R^{2}=0,9028$ entre as taxas obtidas. A diferença nos intervalos é resultante das especificidades de cada método já comentado no tópico materiais e métodos.

Figura 3 - Gráfico comparativo entre EPR e LRR para o trecho analisado

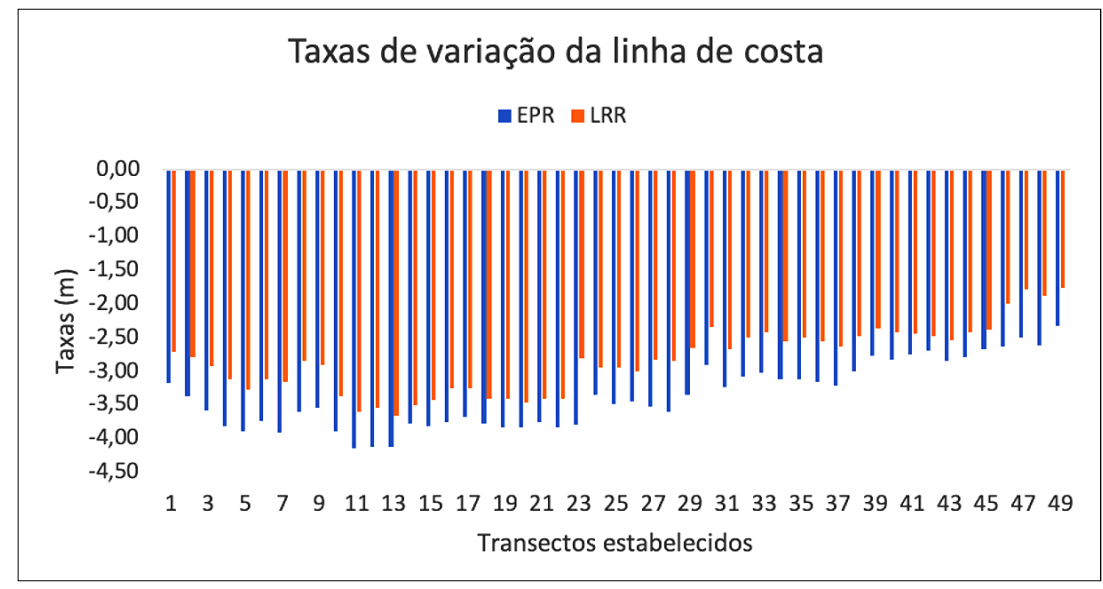

Fonte: os autores.

Figura 4 - Gráfico de correlação entre EPR e LRR para o trecho analisado

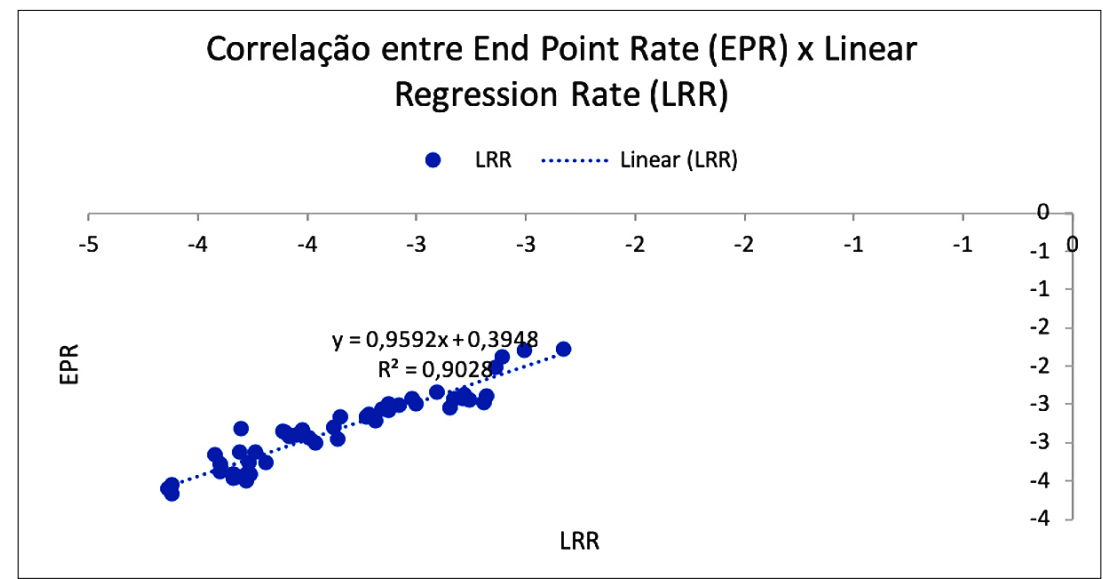

Fonte: os autores.

Os dados do EPR apresentam taxas erosivas que variam entre $-4,14 \mathrm{~m} /$ ano, a máxima, e -2,33 m/ano, a mínima, ou seja, a LC recuou entre -53,82 e -30,29 metros em 13 anos. Podemos perceber, por meio da Figura 5, que tal situação é recorrente com mais intensidade entre os transectos 2 e 29 , trecho entre os pontos 5 e 3, onde a ocupação se faz presente em maior quantidade, principalmente no ponto 3.

O recuo mais intenso da LC nesse setor pode ser resultado da influência direta das formas de ocupação que nesse trecho estão, se não diretamente construídas na LC, muito próximas a ela, o suficiente para promover uma modificação dos processos de transporte de sedimentos, já que entre os transectos 30 e 49 a erosão se apresenta de forma mais amena, em uma área onde não há estruturas que possam barrar a dinâmica natural dos sedimentos. 
Figura 5 - Mapa dos trechos mais vulneráveis à erosão de acordo com o EPR para o trecho analisado da Prainha do Canto Verde

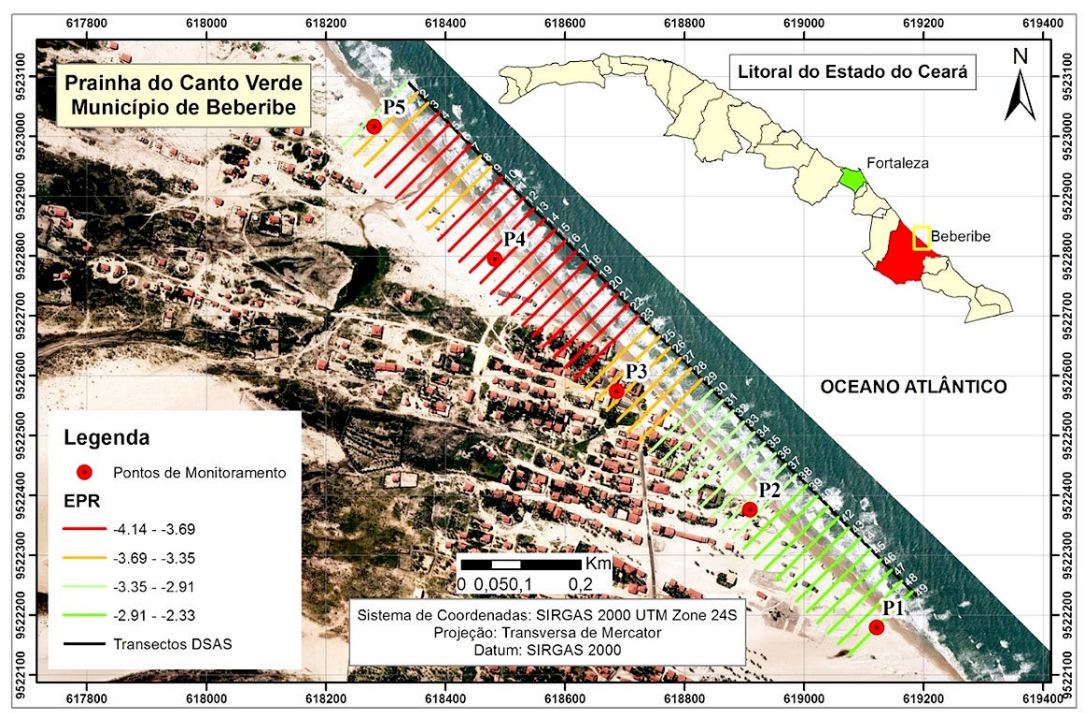

Fonte: os autores.

Diferente do que foi apresentado pelo EPR, os dados obtidos com o LRR mostram taxas de erosão menores, variando entre -3,66 m/ano, o máximo, e -1,77 m/ano, o mínimo, o que representa recuos da LC em alguns trechos na ordem de $-47,58$ a -23,01 metros em 13 anos. Novamente assim como no EPR, os trechos com os maiores recuos estão concentrados na área de influência da ocupação entre os transectos 1 e 28 . É notável que o trecho que merece mais atenção das autoridades e da sociedade civil é o ponto 4, que, mesmo não apresentando formas de ocupações próximas da LC, pode apresentar erosão de dunas frontais que viriam a ser áreas fonte de sedimentos para praias à sotamar desse ponto.

Outro fator que pode influenciar diretamente na erosão desse ponto pode estar diretamente ligado à presença de bancos de areia submersos, o que pode alterar os padrões de direção de onda, aumentado assim o seu potencial erosivo.

Figura 6 - Mapa dos trechos mais vulneráveis à erosão de acordo com o LRR para o trecho analisado da Prainha do Canto Verde

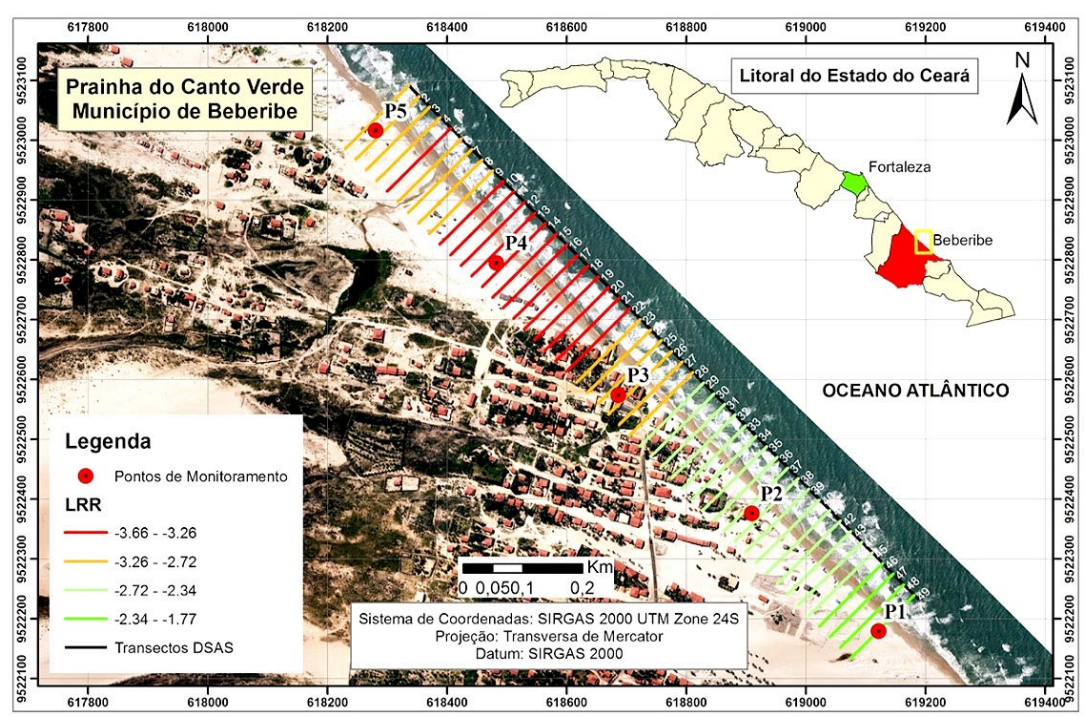

Fonte: os autores. 


\section{CONSIDERAÇÕES FINAIS}

Os resultados obtidos por meio da aplicação da metodologia de análise da variação da linha de costa utilizando o DSAS, seja pelo método EPR, seja pelo LRR, mostram que a erosão se faz presente na área de estudo há pelo menos 13 anos, não havendo qualquer indício de progradação no trecho analisado, e a linha de costa teve um recuo máximo de pouco mais de 50 metros nesse intervalo de tempo. Vale ressaltar que as taxas obtidas mostram que a área apresenta tendências bem próximas ao que já se encontra apresentado na literatura em relação à situação da erosão costeira nas praias do litoral leste do Ceará, mas, por se tratar de um trecho do litoral cearense que ainda não apresenta trabalhos mais específicos, faz-se necessário ainda um monitoramento de campo para análise de médio a longo prazo para assim estabelecer um prognóstico mais conclusivo com relação às perspectivas futuras da região em relação à erosão costeira e seus possíveis danos ao trecho que compreende a Reserva Extrativista da Prainha do Canto Verde.

\section{REFERÊNCIAS BIBLIOGRÁFICAS}

Baptista, P.; Cunha, T.; Bernardes, C.; Gama, C.; Ferreira, O. \& Dias, A. A precise and efficient methodology to analyse the shoreline displacement rate. Journal of Coastal Research, v. 27, n. 2, p. 223-232, 2011.

Barros, E.L. Erosão costeira no município de Icapuí-CE na última década: causas, consequências e perspectivas futuras. 254 f. Tese de doutorado, Programa de Pós-Graduação em Ciências Marinhas Tropicais, Instituto de Ciências do Mar, Universidade Federal do Ceará, Fortaleza, 2018.

Bird, E. Coastal geomorphology: an introduction. Second edition, p. Cm, Jonh Wiley \& Sons, Geostudies, 2008.

Boak, E.H. \& Turner, I.L. Shoreline definition and detection: a review. Journal of Coastal Research, v. 21, n. 4, 2005.

Costa, N.O. de. Cartografia social: instrumento de luta e resistência no enfrentamento dos problemas socioambientais na reserva extrativista marinha da Prainha do Canto Verde, Beberibe Ceará. 2016. 155 f. Dissertação de mestrado, Geografia, Universidade Federal do Ceará, Fortaleza, 2016.

Crowell, M.; Leatherman, S.P. \& Buckley, M.K. Shoreline change rate analysis: long termc versus short term data. Shore and Beach, v. 61, p. 13-20, 1993.

Farinaccio, A. Impactos na dinâmica costeira decorrentes das intervenções em praias arenosas e canais estuarinos de áreas densamente ocupadas no litoral de São Paulo: uma aplicação do conhecimento a áreas não ocupadas. 2008. 218 f. Tese de doutorado, Oceanografia Química e Geológica, Instituto Oceanográfico da Universidade de São Paulo, São Paulo, 2008.

Guerra, R.G. Erosão de praia associada a evolução de esporão arenoso em Parajuru, Beberibe, Ceará. 211 f. Tese de doutorado, Programa de Pós-Graduação em Ciências Marinhas Tropicais, Instituto de Ciências do Mar, Universidade Federal do Ceará, Fortaleza, 2018.

Leatherman, S.P. Shoreline change mapping and management along the u.s. east cost. Journal of Coastal Research, v. 38, p. 5-13, 2003. 
Lins-de-Barros, F.M. Risco, vulnerabilidade física à erosão e impactos sócio-econômicos na orla urbanizada do município de Maricá, Rio de Janeiro. Revista Brasileira de Geomorfologia. União da Geomorfologia Brasileira (UGB), Uberlândia, v. 6, n. 2, p. 83-90, 2005.

Maia, L.P. Processo costeros y balance sedimentario ao lo largo de fortaleza (ne-Brasil): implicaciones para uma gestión adecuada de la zona litoral,1999. 269 p. Tese de doutorado, Universidade de Barcelona, Espanha, 1998.

Mendonça, T.C. de M. Turismo comunitário e pesca: uma relação de complementaridade na Prainha do Canto Verde (Beberibe, CE), in Anais do I Seminário Nacional de Gestão Sustentável de Ecossistemas Aquáticos: Complexidade, Interatividade e Ecodesenvolvimento. Arraial do Cabo, RJ. UFRJ, p. 116-124, 2012.

Morais, J.O.; Pinheiro, L.S.; Cavalcante, A.A.; Paula, D.P. \& Silva, R. L. Erosão costeira em praias adjacentes às desembocaduras fluviais: o caso de pontal de Maceió, Ceará, Brasil. Revista de Gestão Costeira Integrada, v. 8, p. 61-76, 2008.

Morais, J.O.; Pinheiro, L.S.; Freire, G.S.S.; Pessoa, P.R.; Guerra, R.P.; Barros, E.L. \& Moura, J.M. Ceará, in Muehe, D. (org.). Panorama da erosão costeira no Brasil. Brasília: Ministério do Meio Ambiente, p. 261-288, 2018.

Morton, R. A subaerial storm deposits formed on barrier flats by wind-driven currents. Sedimentary Geology, v. 24, p. 105-122, 1979.

Muehe, D. Aspectos gerais da erosão costeira no Brasil. Mercator, Fortaleza, v. 4, n. 7, p. 97-110, 2005.

Muehe, D. Critérios morfodinâmicos para o estabelecimento de limites da orla costeira para fins de gerenciamento. Revista Brasileira de Geomorfologia, v. 2, n. 1, p. 35-44, 2001.

Muehe, D. \& Klum-Oliveira, L. Deslocamento da linha de costa versus mobilidade praial. Quaternary and Environmental Geosciences, v. 5, n. 2, p. 121-124, 2014.

Pinheiro, L.S.; Morais, J.O. \& Maia, L.P. The beaches of Ceará, in Short, A.D.; Klein, A.H.F. (org.). Brazilian beach systems, cap. 7, p. 175-200, Florianópolis: Springer, 2016. (Coastal Research Library 17).

Pontes, E.S.; Silva, E.V. Análise da paisagem: Instrumentos para o turismo comunitário na Prainha do Canto Verde - Ceará. In: Silva, E.V.; Rodriguez, J.M.M.; Meireles, A.J.A. (orgs.) Planejamento Ambiental e Bacias Hidrográficas, p. 41, 2011.

Souza, C.R.G.; Souza Filho, P.W.M.; Esteves, S.L.; Vital, H.; Dillenburg, S.R.; Patchineelam, S.M. \& Addad, J.E. Praias arenosas e erosão costeira, in Souza, C.R.G.; Suguio, K.; Oliveira, A.M.S. \& Oliveira, P.E. de (eds.), Quaternário do Brasil, p. 130-152, Ribeirão Preto: Holos Editora, 2005. ISBN-10: 8586699470.

Thieler, E.R. et al. Digital Shoreline Analysis System (DSAS) versão 4.0: uma extensão do ArcGIS para o cálculo alteração na linha costeira. Serviço Geológico dos EUA Abrir-Arquivo de Relatório 2008-1278, 2009. 